\title{
A multicenter, phase II study of the RAF-kinase inhibitor sorafenib in patients with advanced renal cell carcinoma
}

\author{
HUSSEIN KHALED ${ }^{1}$, HAMDY ABDEL AZIM ${ }^{2}$, EMAD BARSOUM ${ }^{1}$, GEORGE CHAHINE ${ }^{3}$, ALI SHAMSEDDINE ${ }^{4}$, \\ GAMAL ABDEL METAAL $^{5}$, ABBAS OMAR ${ }^{6}$, ABDUL RAHMAN JAZEIH $^{7}$, RASHA HAGGAG $^{8}$ and ATEF BADRAN ${ }^{1}$ \\ ${ }^{1}$ Department of Medical Oncology, National Cancer Institute, Cairo University; ${ }^{2}$ Department of Clinical Oncology, \\ Faculty of Medicine, Cairo University, Cairo 11796, Egypt; ${ }^{3}$ Department of Hematology-Oncology, \\ Hôtel-Dieu de France Hospital; ${ }^{4}$ Department of Internal Medicine, American University of Beirut Medical Center, \\ Beirut 1107 2020, Lebanon; ${ }^{5}$ Department of Medical Oncology, Maadi Military Hospital, Cairo 11796; \\ ${ }^{6}$ Department of Clinical Oncology, Faculty of Medicine, Alexandria University, Alexandria 21131, Egypt; \\ ${ }^{7}$ Department of Oncology, National Guard Hospital, Riyadh 11564, Kingdom of Saudi Arabia; \\ ${ }^{8}$ Department of Medical Oncology, Zagazig University, Sharkia 44519, Egypt
}

Received January 5, 2015; Accepted February 25, 2015

DOI: $10.3892 / \operatorname{mco} .2015 .603$

\begin{abstract}
The treatment of advanced renal cell carcinoma (RCC) has advanced significantly over the last two decades. This multicenter study was designed with the primary objective to evaluate the efficacy and safety of sorafenib as first-line treatment in patients with advanced or metastatic RCC in the Middle East, who were considered to be ineligible for other approved first-line therapies. A total of 75 eligible patients from 8 centers in the Middle East were included in this study. The patients comprised 48 men and 27 women, with a median age of 52 years (range, 19-78 years). A total of 50 patients had clear cell carcinoma, 17 had papillary carcinoma and 8 had other pathological subtypes. At enrollment, 55 of the 75 patients had undergone previous nephrectomy. A total of 67 patients presented with metastatic disease, while 8 patients had regional residual lesions or local recurrence. The patients were treated with $400 \mathrm{mg}$ oral sorafenib twice daily on a continuous basis as a single agent. Treatment was discontinued upon disease progression, prohibitive toxicity, surgical complications, loss to follow-up, or refusal to continue therapy. The median treatment duration was 21 weeks (range, 1-137 weeks). Sorafenib was tolerated by the majority of the patients. Grade 3/4 hand-foot syndrome occurred in 17 patients; diarrhea, elevated liver enzymes and fatigue were observed in 3 patients each; and grade 3/4 vomiting, hypertension and anemia, in 1 patient each. Of the 75 patients included in this study, 60 were evaluable for response. One patient achieved a complete response for 91 weeks and 6 patients exhibited
\end{abstract}

Correspondence to: Professor Hussein Khaled, Department of Medical Oncology, National Cancer Institute, Cairo University, Fom El Khalig Square, Cairo 11796, Egypt

E-mail: khussein528@gmail.com

Key words: renal cell carcinoma, sorafenib, phase II study a partial response (median duration of 23 weeks) with an overall response rate of $11.7 \%$. Disease stabilization occurred in 37 patients $(61.7 \%)$. Thus, disease control was achieved in 44 of the 60 patientrs $(73 \%)$. At a median follow-up period of 53.5 weeks (range, 8.5-192 weeks), an intention-to-treat analysis demonstrated a median time-to-disease progression of 25.7 weeks, with a median overall survival of 54.8 weeks. In conclusion, sorafenib was found to be tolerable and effective as first-line therapy in patients with advanced RCC.

\section{Introduction}

Renal cell carcinoma (RCC) represents $2.4 \%$ of all malignancies worldwide (http://globocan.iarc.fr/Pages/fact_ sheets_cancer.aspx). In addition, RCC accounts for $90 \%$ of renal tumors, with $85 \%$ of RCCs being of the clear cell type (1). According to the Surveillance of Epidemiology and End Results data from the USA, the 5-year survival rate of advanced renal cancer is $\sim 12.6 \%$ (http://seer.cancer.gov/statfacts/html/kidrp.html). Cytoreduction surgery followed by systemic therapy is generally recommended for patients with advanced disease $(2,3)$. Tyrosine kinase inhibitors are widely used as first- and second-line treatment and, to date, 7 such agents, including sorafenib, have been approved by the Food and Drug Administration for the treatment of advanced RCC [National Comprehensive Cancer Network (NCCN) guidelines 2014, www.nccn.com].

Sorafenib tosylate is a small molecule that inhibits multiple serine/threonine kinases, RAF and other receptor kinases, e.g., vascular endothelial growth factor receptor (VEGFR)-1, -2 and -3 , platelet-derived growth factor receptor- $\beta$ and other receptors (4-8). While this drug was considered as a standard of care for patients who progressed on prior cytokine therapy (9-11) or following treatment with a tyrosine kinase inhibitor $(12,13)$, only one randomized phase II clinical study (14) has been published on using sorafenib as first-line therapy. 
Table I. Lesions of the 75 patients at presentation.

Lesions

Randomized patients $(\mathrm{n}=75)$

Evaluable patients $(\mathrm{n}=60)$

\section{Residual mass}

Local recurrence

Metastatic lung lesions only

Metastatic liver lesions only

Metastatic lymph node only

Multiple organ metastasis

(lung, liver, bone, lymph nodes)
4

4

15

3

3

46
3

3

13

2

2

37
Thus, the aim of this multicenter study was to confirm the efficacy and evaluate the safety of sorafenib as first-line therapy in patients with advanced or metastatic RCC.

\section{Patients and methods}

Patient selection. A total of 75 eligible patients from 8 centers in 3 Middle East countries (Egypt, Lebanon and Saudi Arabia) were recruited.

The eligibility criteria included pathologically proven advanced RCC, Eastern Cooperative Oncology Group performance status 0-2 and measurable disease. The patients were administered sorafenib as first-line therapy, as they were considered ineligible to receive other approved first-line therapies and, in the investigator's opinion, they were reasonably likely to benefit from single-agent sorafenib treatment.

Adequate renal, hepatic and bone marrow function was required, as defined by a serum creatinine $\leq 2.0 \mathrm{x}$ institutional upper limit of normal (ULN), total bilirubin $\leq 1.5 \times$ ULN, aspartate aminotransferase/alanine aminotransferase $\leq 2.5 \times \mathrm{ULN}$, leukocyte count $\geq 3,000 / \mu 1$, absolute neutrophil count $\geq 1,500 / \mu 1$ and platelet count $\geq 75,000 / \mu 1$. The patients provided written informed consent prior to receiving sorafenib.

The exclusion criteria included serious cardiovascular disease, a life expectancy of $<2$ months, metastatic brain or meningeal tumors, recent or active bleeding diathesis and any prior systemic therapy for RCC.

The study protocol was approved by the review boards of all the participating institutions. All the procedures were conducted according to the principles of the Declaration of Helsinki.

Baseline evaluation. The baseline patient evaluation included medical history and physical examination, laboratory investigations (complete blood count and blood chemistry) and tumor radiological imaging, including computed tomography (CT) scan or magnetic resonance imaging (MRI) of the chest, abdomen and pelvis, isotope bone scan and brain MRI or CT scan.

Treatment protocol. The treatment was administered as $400 \mathrm{mg}$ sorafenib orally, twice daily, continuously in 4-week cycles. Dose increases were not permitted, while dose reductions to $400 \mathrm{mg}$ daily and then every other day was allowed, depending on the type and severity of toxicity encountered and provided that the criteria for patient withdrawal from the study treatment were not met. The patients continued to receive treatment with sorafenib until development of intolerable toxicity, disease progression, or withdrawal of consent for any reason.

Response to treatment and adverse events. Tumor assessments were performed according to the Response Evaluation Criteria in Solid Tumors at baseline and every 8 weeks thereafter, or earlier if tumor progression was clinically suspected (15). Any adverse events were reported and graded according to the Common Toxicity Criteria, version 4.0 [National Cancer Institute. Common Terminology Criteria for Adverse Events (CTCAE) Version 4.0. http://evs.nci.nih.gov/ftp1/CTCAE/CTCAE_4.03_201006-14_QuickReference_5x7.pdf]. All the responses were reviewed by independent experts at the study completion, including simultaneous review of patient files and radiological images.

Statistical analysis. Patient demographic data and baseline characteristics were analyzed by summary statistics for quantitative variables and frequency tables for qualitative variables. Efficacy data were summarized by frequency tables. Overall survival was defined as the time from randomization to death from any cause; progression-free survival was calculated from the date of first administration of sorafenib to the investigator's assessment or radiological documentation of disease progression. Survival was analyzed by the Kaplan-Meier estimate. Statistical analysis was performed using IBM SPSS software, version 20 (IBM SPSS, Chicago, IL, USA).

\section{Results}

Participating institutions. Between October, 2008 and February, 2011, 75 eligible patients were recruited to participate in this study. Of the 75 patients, 65 were from Egypt, 8 from Lebanon and 2 from Saudi Arabia. The majority of the Egyptian patients were recruited from the National Cancer Institute, Cairo University $(n=43)$, while the remaining 22 patients were recruited from four additional centers (two private centers, the Clinical Oncology Department of Alexandria University and the Maadi Military Hospital). Two centers in Lebanon participated in the study (American University in Beirut, 3 patients; and Hôtel-Dieu de France, 5 patients). The remaining 2 patients were recruited from the National Guard Hospital in Saudi Arabia. 
Table II. AEs according to the CommonToxicity Criteria v.4.0.

\begin{tabular}{lccccc}
\hline & \multicolumn{2}{c}{ All patients } & & \multicolumn{2}{c}{ Evaluable } \\
\cline { 2 - 3 } \cline { 5 - 6 } Symptoms & Grade 3 & Grade 4 & & Grade 3 & Grade 4 \\
\hline Pain & 2 & 0 & & 2 & 0 \\
Fatigue & 2 & 1 & & 0 & 1 \\
Hand-foot & 16 & 1 & & 16 & 1 \\
syndrome & & & & & \\
Elevated & 3 & 0 & & 0 \\
liver enzymes & & & & & 0 \\
Vomiting & 2 & 0 & & 3 & 0 \\
Diarrhea & 3 & 0 & & 1 & 0 \\
Anemia & 1 & 0 & & 1 & 0 \\
Hypertension & 1 & 0 &
\end{tabular}

AEs, adverse events.

Patient characteristics. The patients comprised 48 men and 27 women, with a median age of 52 years (range, 19-78 years). A total of 50 patients had clear cell carcinoma, 17 had papillary carcinoma and 8 had other pathological subtypes (5 chromophobe, 1 medullary and 2 unclassified RCCs). The majority of the patients $(\mathrm{n}=55 ; 73 \%)$ had undergone prior nephrectomy.

A total of 67 patients presented with metastatic disease, while 8 patients presented with regional residual lesions or local recurrence. Multiple organ metastases (lung, liver, bone and lymph nodes) were present in 46 patients, while only lung, liver, or lymph node metastases were found in 15 , 3 and 3 patients, respectively (Table I).

Adverse events. The duration of sorafenib treatment was 1-137 weeks, with a median duration of 21 weeks. The most commonly reported grade $3 / 4$ adverse events were hand-foot syndrome, fatigue, diarrhea, elevated liver enzymes, vomiting and generalized pain. Anemia and hypertension occurred with a lower frequency (Table II). Certain adverse events were severe enough to require hospitalization (11 patients).

Response. Of the 75 patients included in the study, 15 were non-evaluable for treatment response due to the patient's wish to discontinue treatment $(\mathrm{n}=5)$, death prior to evaluation $(n=4)$, serious adverse event and/or drug toxicity $(n=3)$, refusal to initiate treatment following enrollment $(n=2)$ and non-compliance $(\mathrm{n}=1)$.

During a median observation time of 53.5 weeks (range, 8.5-192 weeks), 1 patient achieved a complete response and 6 patients had partial remissions, with an overall response rate of $11.7 \%$ (7/60 patients). The duration of the complete remission was 91 weeks, while the partial remission durations ranged between 9 and 35 weeks, with a median of 23 weeks. Additionally, 37 patients (61.7\%) exhibited disease stabilization. Thus, disease control was achieved in $44 / 60$ patients (73\%). The disease control rate was not affected by pathological subtype (clear cell vs. non-clear cell), being $68.4 \%$ (26/38 patients) and $81.8 \%$ (18/22 patients), respectively $(\mathrm{P}=0.52)$.

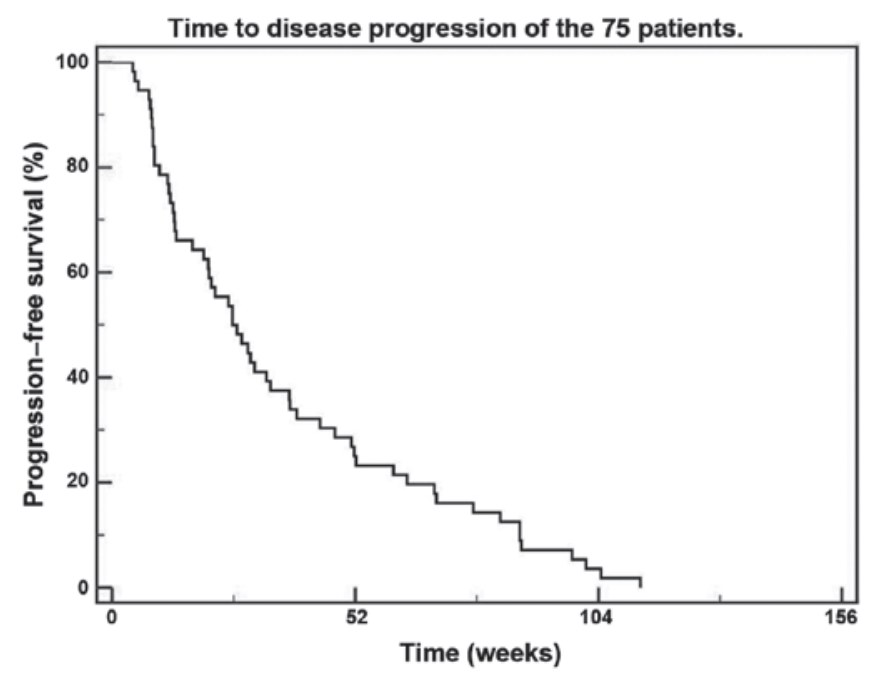

Figure 1. Time to disease progression of the 75 patients.

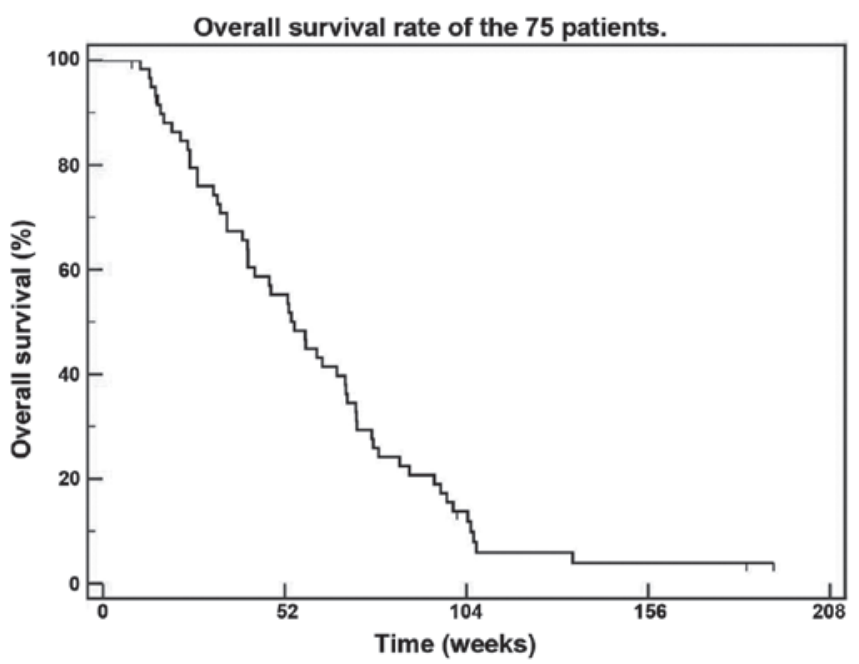

Figure 2. Overall survival rate of the 75 patients.

At the time of study completion, 6 patients remained alive. In an intention-to-treat analysis, the median time-to-disease progression (TDP) was 25.7 weeks (Fig. 1), while the median overall survival was 54.8 weeks (Fig. 2). The median TDP and the median overall survival were not affected by the pathological subtype, being 25.7 and 53.1 weeks for the clear cell type $(\mathrm{P}=0.1)$ and 38 and 66.9 weeks for the non-clear cell subtypes $(\mathrm{P}=0.8)$, respectively.

\section{Discussion}

The systemic treatment options for advanced RCC were, until recently, limited to cytokine therapy, e.g., interferon and interleukin-2. Targeted therapies, mainly with tyrosine kinase inhibitors, were introduced over the last few years. These include agents such as sunitinib, sorafenib, axitinib, temsirolimus, pazopanib, everolimus and bevacizumab. Tumor histology and risk factor evaluation are crucial for selecting the targeted agent suitable for each patient. 
Interim data from the phase III TARGET trial on advanced RCC demonstrated a significant increase, with near doubling of the progression-free survival for patients receiving sorafenib vs. placebo (5.5 vs. 2.8 months, respectively). That trial included $>900$ patients who exhibited disease progression following prior therapy (9). This encouraged investigators to evaluate the effects of sorafenib as first-line treatment for patients with advanced RCC $(14,16,17)$.

The present study demonstrated that sorafenib, administered as first-line treatment for patients with advanced RCC, is tolerable and effective. A previous randomized study by Escudier et al (14) provided the first evidence that sorafenib may be administered as front-line therapy, with a tumor shrinkage rate of $68.2 \%$ and a median progression-free survival of 5.7 months. These figures are comparable to those of the present study, leading NCCN to list sorafenib as a category $2 \mathrm{~A}$ option for the first-line therapy of advanced RCC (NCCN guidelines 2014, www.nccn.com). The progression-free survival rate with other targeted agents, such as sunitinib, pazopanib and temsirolimus, in the front-line setting was reportedly 11.9, 2 and 5.5 months, respectively, with an overall objective response rate of $\sim 30 \%$ (NCCN guidelines 2014, www.ncen.com).

The observed clinical activity of the sequential use of VEGF inhibitors indicates persistent RCC tumor reliance on VEGF signaling following exposure to different VEGF-binding or other VEGFR inhibitors. Therefore, response or lack of response to prior VEGF-targeted therapy does not appear to affect further therapy with agents exhibiting similar mechanisms of action. Iacovelli et al (18) recently reported data from a cohort of patients who had received three lines of therapy, suggesting that the sequence of VEGF inhibitor followed by another VEGF inhibitor followed by a mechanistic target of rapamycin (mTOR) inhibitor may be associated with better survival compared with a sequence in which an mTOR inhibitor was sandwiched between two VEGF inhibitors. Phase III trials are required to further support this concept and may help define an optimal sequence on an individual patient basis.

There were certain limitations to the present study. First, this was a single-agent, non-randomized study. Therefore, the relative benefit of sorafenib as compared with other agents was not clearly determined. Second, there are certain inherent limitations to response evaluation criteria, particularly radiological evaluation, with targeted agents in RCC, including treatment with sorafenib.

In conclusion, the present study demonstrated that the use of first-line sorafenib for patients with advanced RCC is a tolerable and effective treatment option.

\section{References}

1. Karumanchi SA, Merchan J and Sukhatme VP: Renal cancer: Molecular mechanisms and newer therapeutic options. Curr Opin Nephrol Hypertens 11: 37-42, 2002.

2. Flanigan RC, Salmon SE, Blumenstein BA, Bearman SI, Roy V, McGrath PC, Caton JR Jr, Munshi N and Crawford ED: Nephrectomy followed by interferon alfa-2b compared with interferon alfa-2b alone for metastatic renal-cell cancer. N Engl J Med 345: 1655-1659, 2001.
3. Choueiri TK, Xie W, Kollmannsberger C, North S, Knox JJ, Lampard JG, McDermott DF, Rini BI and Heng DY: The impact of cytoreductive nephrectomy on survival of patients with metastatic renal cell carcinoma receiving vascular endothelial growth factor targeted therapy. J Urol 185: 60-66, 2011.

4. Awada A, Hendlisz A, Gil T, et al: Phase I safety and pharmacokinetics of BAY 43-9006 administered for 21 days on/7 days off in patients with advanced, refractory solid tumours. Br J Cancer 92: 1855-1861, 2005.

5. Clark JW, Eder JP, Ryan D, Lathia C and Lenz HJ: Safety and pharmacokinetics of the dual action Raf kinase and vascular endothelial growth factor receptor inhibitor, BAY 43-9006, in patients with advanced, refractory solid tumors. Clin Cancer Res 11: 5472-5480, 2005.

6. Moore M, Hirte HW, Siu L, Oza A, Hotte SJ, Petrenciuc O, Cihon F, Lathia C and Schwartz B: Phase I study to determine the safety and pharmacokinetics of the novel Raf kinase and VEGFR inhibitor BAY 43-9006, administered for 28 days on/7 days off in patients with advanced, refractory solid tumors. Ann Onco 16: 1688-1694, 2005.

7. Strumberg D, Richly H, Hilger RA, et al: Phase I clinical and pharmacokinetic study of the Novel Raf kinase and vascular endothelial growth factor receptor inhibitor BAY 43-9006 in patients with advanced refractory solid tumors. J Clin Oncol 23: 965-972, 2005.

8. Wilhelm SM, Carter C, Tang L, et al: BAY 43-9006 exhibits broad spectrum oral antitumor activity and targets the RAF/MEK/ERK pathway and receptor tyrosine kinases involved in tumor progression and angiogenesis. Cancer Res 64: 7099-7109, 2004

9. Escudier B, Eisen T, Stadler WM, et al; TARGET Study Group: Sorafenib in advanced clear-cell renal-cell carcinoma. N Engl J Med 356: 125-134, 2007.

10. Eisen T, Bukowski RM, Staehler M, Szczylik C, Oudard S, Stadler WM, Schwartz B, Simantov R, Shan M and Escudier B; The Sorafenib TARGETs Clinical Trial Group: Randomized phase III trial of sorafenib in advanced renal cell carcinoma (RCC): Impact of crossover on survival. 2006 ASCO Annual Meeting Proceedings. J Clin Oncol 24 (Suppl 18): 4524, 2006.

11. Bukowski RM, Eisen T, Szczylik C, Stadler WM, Simantov R, Shan M, Elting J, Pena C and Escudier B: Final results of the randomized phase III trial of sorafenib in advanced renal cell carcinoma: Survival and biomarker analysis. ASCO Annual Meeting Proceedings (Post-Meeting Edition). J Clin Oncol 25 (Suppl 18): 5023, 2007.

12. Di Lorenzo G, Cartenì G, Autorino R, et al: Phase II study of sorafenib in patients with sunitinib-refractory metastatic renal cell cancer. J Clin Oncol 27: 4469-4474, 2009.

13. Garcia JA, Hutson TE, Elson P, Cowey CL, Gilligan T, Nemec C, Dreicer R, Bukowski RM and Rini BI: Sorafenib in patients with metastatic renal cell carcinoma refractory to either sunitinib or bevacizumab. Cancer 116: 5383-5390, 2010.

14. Escudier B, Szczylik C, Hutson TE, et al: Randomized phase II trial of first-line treatment with sorafenib versus interferon alfa-2a in patients with metastatic renal cell carcinoma. J Clin Oncol 27: 1280-1289, 2009.

15. Therasse P, Arbuck SG, Eisenhauer EA, et al: New guidelines to evaluate the response to treatment in solid tumors. European Organization for Research and Treatment of Cancer, National Cancer Institute of the United States, National Cancer Institute of Canada. J Natl Cancer Inst 92: 205-216, 2000.

16. Park SJ, Lee JL, Park I, et al: Comparative efficacy of sunitinib versus sorafenib as first-line treatment for patients with metastatic renal cell carcinoma. Chemotherapy 58: 468-474, 2012.

17. Procopio G, Derosa L, Gernone A, et al: Sorafenib as first- or second-line therapy in patients with metastatic renal cell carcinoma in a community setting. Future Oncol 10: 1741-1750, 2014.

18. Iacovelli R, Cartenì G, Sternberg CN, et al: Clinical outcomes in patients receiving three lines of targeted therapy for metastatic renal cell carcinoma: Results from a large patient cohort. Eur J Cancer 49: 2134-2142, 2013. 